\title{
Yeast biodiversity from Vitis vinifera L., subsp. sylvestris (Gmelin) Hegi to face up the oenological consequences of climate change
}

\author{
Anna Puig-Pujol ${ }^{1}$, Núria Ferrando ${ }^{1}$, Fina Capdevila ${ }^{1}$, Rafael Ocete $^{2}$, and Eugenio Revilla ${ }^{3}$ \\ ${ }^{1}$ Department of Enological Research, Institute of Agrifood Research and Technology-Catalonian Institute of Vine and Wine \\ (IRTA-INCAVI), Plaça Àgora 2, 08720 Vilafranca del Penedès, Barcelona, Spain \\ ${ }^{2}$ Faculty of Biology, University of Sevilla. Avda. de la Reina Mercedes, s/n, 41012 Sevilla, Spain \\ ${ }^{3}$ Department of Agricultural Chemistry and Food Science, Autonomous University of Madrid, 28049 Madrid, Spain
}

\begin{abstract}
The impact of climate change in the viticulture is affecting the quality of grapes and their wines. As consequence, climatic variations are producing a mismatch between technological and phenolic maturity and are affecting the microbiota's ecology, biodiversity and their metabolism in vineyard, grape, must and wine. However, there are natural resources that can help to mitigate the effects of global warming. It has been noticed that grapes from female plants of wild vines (Vitis vinifera subsp. sylvestris) have very appropriate characteristics to face up this problem: later maturing, high acidity, high polyphenol content,... A molecular study of 819 strains isolated at the end of spontaneous fermentations of grapes of Vitis vinifera subsp. sylvestris grapevines from 30 locations in northern of Spain revealed 8 different genera and 18 different species. 71,5\% of the yeasts were classified as non-Saccharomyces and 28,5\% were identified as Saccharomyces cerevisiae. This latter specie was characterized at strain level, classifying 30 different groups, 6 of which as the majority from 2 up to 4 different locations. These findings demonstrate a wide diversity of yeast microbiota in wild grapes that will allow a yeast selection for the wine industry in a scenario of climate change.
\end{abstract}

\section{Introduction}

There is an almost global agreement within the scientific community that climate change is a fact that today cannot be ignored. Climate change is exerting an increasingly profound influence on vine phenology and grape composition and ultimately affects vinifications, wine microbiology and chemical and sensory aspects [1]. From the point of view of the plant, global warming will involve a major water shortage and high salinity in soil that might affect their growth. Concerning oenological microbiology, forecasted variations in the environmental conditions (temperature, water stress...), in the composition of the substrate (higher grape berry sugar, lower acidity and higher $\mathrm{pH}$ values) are likely to affect the microbiota's ecology, biodiversity and their metabolism [2]. Natural genetic diversity present in the grapevine allows to look for new genotypes (plants and microorganisms) adapted to new climate conditions. This might be an appropriate strategy to cope with changes generated by climate change.

Related to this fact, wild grapevines represent a unique, invaluable genetic resource for cultivated grapevines. Vitis vinifera subsp.sylvestris is the wild subspecies of $V$. vinifera $\mathrm{L}$. wich includes the largely cultivated $V$. vinifera subsp. vinifera. The species disperses seeds through birds and colonised a broad range of habitats and soils in the Mediterranean basin [3]. In Spain, natural populations of $V$. vinifera ssp. sylvestris can still be found along river banks and coasts [4]. It has been described some interesting oenological and phenotypic characteristics of grapes from these populations such as high acidity and low $\mathrm{pH}$ and a high intensity of colour $[5,6]$. These features might be used to mitigate the potential effects of global warming on grape production, using a natural resource to adapt the Viticulture to the new climate conditions.

As far as we know, few or not studies have been published about yeast microflora that is present on $V$. vinifera ssp. sylvestris. The aim of this work has been to analyse the yeast biodiversity in this type of wild grapes in order to select indigenous strains and create a yeast collection that could allow cellars to produce wines using new yeasts and varieties adapted to climate change.

\section{Materials and methods}

V. vinifera L. ssp. sylvestris grape samples were harvested from female vines in 24 locations of the North of Spain during October and November, 2015 (Fig. 1).

The grapes were collected in each location in sterile bags and transported to the laboratory. Black berries were crushed under aseptic conditions using a stomacher machine.

Fermentations were carried out with indigenous yeasts, with skin maceration and without addition of sulphur dioxide, at $22^{\circ} \mathrm{C}$. The kinetics of the process was monitored by measuring the residual sugar. When it was less than $2 \mathrm{~g} / \mathrm{L}$ (final fermentation), sample was taken for yeasts isolation. 
Serial diluted samples (in Ringer solution) of each fermentation were plated on Wallerstein Laboratory Nutrient (WLN) medium (Oxoid), which is able to differentiate yeast populations on the basis of the colour and morphology of the colony [7]. Plates were incubated at $28^{\circ} \mathrm{C}$ for 4 days. After counting yeast concentration, at least 20 colonies were selected and isolated on the basis of their appearance. Streaking was carried out on WLN and pure cultures were stored at $4{ }^{\circ} \mathrm{C}$ until molecular analysis.

For the molecular analysis, two different approaches were carried out in order to identify yeasts at genus or specie level [8]. Prior to identification, genomic DNA of each isolate was extracted from $1,8 \mathrm{ml}$ of an overnight culture in YPD broth, according to Querol et al. [9]. First, all isolates were analyzed by restriction fragment length polymorphism (RFLP) of the region ITS1-5.8S rRNAITS2. The DNA fragments were amplified using primers ITS 1 and ITS4, and PCR conditions were carried out as described by Esteve-Zarzoso et al. [10]. The amplified fragments were digested with endonucleases HinfI and HaeIII (New England Biolabs), according to the supplier's instructions. The DdeI enzyme was adopted in order to discriminate species of the genus Hanseniaspora [10]. Both PCR products and restriction fragments were separated by electrophoresis in 1.4 and $3.0 \%$ agarose gels respectively and stained with ethidium bromide (Sigma). The approximate lengths were determined by the use of molecular weight ladder (100 bp, Roche). PCR and RFLP fragment lengths were used for identification of yeasts by comparison of the restriction bands with those available in literature [10-13]. Several groups appeared on the basis of their RFLP of the ITS region.

Yeast identification was confirmed by analyzing the D1/D2 region of the 26S rRNA gene. At least two strains of each group were amplified with NL1 and NL4 primers, as reported by Kurtzman and Robnett [14]. PCR procedure and subsequent visualized steps were done as described by Wang and Liu [15]. PCR amplicons were purified by the QIAquick PCR purification kit (Qiagen) and were sent to a commercial facility (Secugen, Madrid, Spain) for sequencing, using the primer NL1 employed for PCR amplification. Resulting sequences were aligned in GeneBank database using the Blast method of NCBI (http://www.ncbi.nlm.nih.gov/blast).

In order to be identified at strain level, interdelta sequence amplifications of all Saccharomyces cerevisiae isolates were carried out using delta 12 and delta 21 primers according to Legras and Karst [16]. The PCR products were separated by electrophoresis on $2.0 \%$ agarose gels, which was applied with $100 \mathrm{~V}$ for $3 \mathrm{~h}$ in 1x TBE buffer, stained with ethidium bromide and photographed under UV light. Different patterns (strains) were obtained by comparing the bands distribution of each S. cerevisiae.

\section{Results and discussion}

The consequences of global warming in Viticulture, with advanced harvest times, increased grape sugar concentrations, lower acidities and higher $\mathrm{pH}$ values involves changes in microbial ecology of musts and wines and increase the risk of spoilage and organoleptic degradation. For this reason, there is a growing demand for new and improved wine yeast strains adapted to these

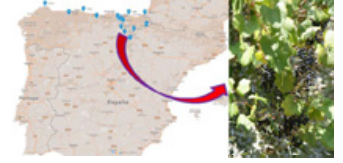

Figure 1. Locations where grapes were collected.

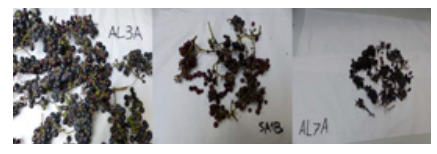

Figure 2. Examples of the size of the samples and grapes.

Table 1. Range of main oenological parameters in musts of $V$. vinifera L. sylvestris used for yeast isolation.

\begin{tabular}{|l|c|c|}
\hline Variable & Minimum value & Maximum value \\
\hline $\begin{array}{l}\text { Probable alcoholic } \\
\text { degree }(\% \text { vol })\end{array}$ & 5.3 & 13.2 \\
\hline Gluc + Fruct $(\mathrm{g} / \mathrm{L})$ & 44.8 & 222 \\
\hline $\begin{array}{l}\text { Titratable acidity } \\
\text { g/L tartaric acid) }\end{array}$ & 5.6 & 27.7 \\
\hline Malic acid (g/L) & 2.7 & 21.7 \\
\hline pH & 2.91 & 3.80 \\
\hline Acetic acid (g/L) & 0.10 & 0.34 \\
\hline $\begin{array}{l}\text { Gluconic acid } \\
\text { g/L) }\end{array}$ & $<0.1$ & 0.8 \\
\hline
\end{tabular}

new conditions. The results of the present study show a first approach to the yeast diversity that can be found in a natural resource as wild vines ( $V$. vinifera $L$. ssp. sylvestris), that have interesting oenological traits that could be used to counteract the effects of climate-change related variables.

Grapes were collected at 30 locations in the North of Spain (Fig. 1). Due to the different soil and climatic conditions of these environments, the maturity of bunches were variable, an inherent characteristic of wild grapevines populations [5]. The number of bunches per plant and the berries per bunch was also highly variable. Red berries had a small size, with a diameter less than $1 \mathrm{~cm}$ (Fig. 2). As a consequence, the weight of the samples and the amount of the must obtained were also different in each location: it ranged from $44 \mathrm{~g}$ to $560 \mathrm{~g}$.

A summary of the main oenological variables of the musts where yeasts were isolated are shown in Table 1.

Fermentation process at $22^{\circ} \mathrm{C}$ was performed between 5 and 26 days, depending on the batch of must. During this period yeasts population reached concentrations up to $2.7 \cdot 10^{8} \mathrm{cfu} / \mathrm{ml}$.

A total of 819 purified colonies isolated from the end of the 30 fermentations of sylvestris grape samples were subjected to PCR-RFLP analysis of the 5.8SITS rDNA region. PCR products varying in length from 390 to $880 \mathrm{bp}$ were digested with HinfI, HaeIII and DdeI enzymes, and the molecular mass of the restriction products obtained was compared with those described previously in the literature [10-13]. In general, we observed 18 different profiles of the ITS-5.8S rDNA region. Two randomly selected strains for each pattern obtained were analysed by the D1/D2 region of the 26S rRNA gene and sequenced to confirm species assignation, performed by comparison with sequences available at the NCBI database (GenBank) using the standard nucleotide_nucleotide homology 
Table 2. Percentage frequencies and $\mathrm{n}^{\circ}$ of locations where Vitis vinifera $\mathrm{L}$ ssp sylvestris yeast species were isolated.

\begin{tabular}{|l|c|c|}
\hline Specie & Frequency $\mathbf{\%}$ ) & $\mathbf{N}^{\circ}$ of locations \\
\hline Hanseniaspora uvarum & 44.08 & 17 \\
\hline Saccharomyces cerevisiae & 28.57 & 15 \\
\hline Candida californica & 7.69 & 5 \\
\hline Pichia anomala & 4.76 & 4 \\
\hline Hanseniaspora valbyensis & 4.52 & 4 \\
\hline Pichia manshurica & 2.81 & 2 \\
\hline Lachancea thermotolerans & 1.71 & 3 \\
\hline Hanseniaspora osmophila & 1.47 & 2 \\
\hline Zygosaccharomyces bailii & 1.34 & 1 \\
\hline Pichia membranifaciens & 0.98 & 1 \\
\hline Pichia kudriavzevii & 0.85 & 1 \\
\hline Kluyveromyces marxianus & 0.37 & 1 \\
\hline Metschnikowia fructicula & 0.24 & 2 \\
\hline Hanseniaspora vineae & 0.12 & 1 \\
\hline Kloeckera lindneri & 0.12 & 1 \\
\hline Lachancea fermentati & 0.12 & 1 \\
\hline Pichia occidentalis & 0.12 & 1 \\
\hline $\begin{array}{l}\text { Zygosaccharomyces } \\
\text { florentinus }\end{array}$ & 0.12 & 1 \\
\hline
\end{tabular}

search Basic Local Alignment Search Tool (BLAST, (http://www.ncbi.nlm.nih.gov/blast). The yeast species identified and their isolation frequencies obtained at the end of spontaneous fermentations and the number of different locations where they were found is shown in Table 2.

\section{Non-Saccharomyces yeast biodiversity}

A wide variety of non-Saccharomyces yeasts were found: $71.5 \%$ of the strains analysed, that were included in 8 genera and 17 different species. The most abundant was $H$. uvarum, isolated in more or less frequency in 17 of the 30 fermentations analysed. Three additional Hanseniaspora species: $H$. valbyensis, $H$. osmophila and $H$. vineae and the related species Kloeckera lindneri [17] were detected in the wines. The apiculate yeasts represent often the dominant microbial population of the early stages of grape must fermentation. Even if the growth of these yeasts is limited only to the first days of fermentation, the activity of Hanseniaspora (Kloeckera) wine strains can influence significantly the chemical composition of the final wine. Although $H$. uvarum is known as high producer of acetic acid [18], the large variability among $H$. uvarum wine strains, could allow the identification of strains suitable to be used as starter culture in mixed fermentation with S. cerevisiae [19]. Several studies have shown the impact of Hanseniaspora species on the analytical composition and sensory characteristics of wine. Viana et al. [20] suggested the potential of using $H$. osmophila in mixed cultures with $S$. cerevisiae to increase the levels of 2-phenylethyl acetate (fruity and flowery aroma) in wines. And similar results were obtained by Viana et al [21] and Lleixà et al [22] when strains of $H$. vineae were used in co-culture with $S$. cerevisiae. Although in this study samples for yeasts isolation were taken at the end of fermentation, when concentration of residual sugars was less than $2 \mathrm{~g} / \mathrm{L}$, the alcohol content in all samples where Hanseniasposa sp. was detected was low (less than $5 \%$ vol). Thus could explain the high frequency of presence of these species.
Other genus quite abundant in grapes of $V$. vinifera L. ssp sylvestris is Pichia sp. We identified 5 different species $(9.52 \%$ of the total isolates), some of them in low ratio (Table 2): P. anomala (Hansenula anomala or Wickerhamomyces anomalus), P. manshurica, P. membranifaciens, $P$. kudriavzevii and $P$. occidentalis (Issatchenkia occidentalis). P. anomala and P. membranifaciens have been suggested for the production of lowalcohol wines [23] and some strains of $P$. anomala have $\beta$-glucosidase activity with aroma-enhancing capabilities in wine [24]. And recently $P$. kudriavzevi has been described as representative yeast of North Patagonian winemaking terroir [25].

The genus Zygosaccharomyces sp. included two species: Z. bailii and Z. florentinus were detected in two spontaneous fermentations, with $1,34 \%$ and $0.12 \%$ of presence, respectively. Some literature considers this genus to be winery contaminants producing high quantities of acetic acid and is specially a problem in wineries producing sweet and sparkling wines due to its capacity to referment bottled wines. However, it has been described that some Z. bailii strains produces high levels of ethyl esters as a mixed starter with $S$. cerevisiae, increasing the aromatic complexity of wine [26].

A species isolated in this work and also relevant in technological terms is Lachancea thermotolerans (Kluyveromyces thermotolerans), found in $1.71 \%$ frequency in 3 different locations. This yeast has the interesting property of being able to metabolize sugar into lactic acid and thereby decreasing the level of ethanol produced from grape must and enhance acidity [27,28].

Kluyveromyces marxianus were isolated three times, representing $0.37 \%$ of the yeasts. This species is able to ferment sugars in high temperature environments (up to $45^{\circ} \mathrm{C}$ ), including grape juice. It is also present in some commercial preparations to contribute flavour complexity [29].

Two strains of Metschnikowia fructicola $(0.24 \%$ of frequency), close relative of $M$. pulcherrima, were found in two distinct locations. It has been described to possess antifungal activity, suggesting that the presence of Metschnikowia species, specifically $M$. pulcherrima and $M$. fructicola may play a protective role against the growth of filamentous fungi on grapes during withering [30].

Although detected in fairly high frequency $(7.69 \%)$ in 5 different locations, no relevant information related to improve wine quality have been found for Candida californica.

Thus, in general, wine makers and wine researchers have come to realize that non-Saccharomyces yeasts contribute in significant measure to the flavour and quality of wine than previously thought. Currently, fermentation trials are performed with all the species isolated from wild grapes to find someone that exhibits promising phenotypic traits suitable for wine-making.

\section{Saccharomyces cerevisiae strains community in wild grapes}

$S$. cerevisiae is the most important yeast for wine production and the conversion of sugar in ethanol, it being the main yeast at the end of spontaneous fermentation and also as commercial starter culture [31]. 
Table 3. Classification of $S$. cerevisiae strains and locations where they were isolated.

\begin{tabular}{|c|c|c|}
\hline $\begin{array}{c}\text { S. cerevisiae } \\
\text { genotypes }\end{array}$ & $\begin{array}{c}\text { Location } \\
\text { (n isolates/location) }\end{array}$ & $\begin{array}{c}\text { Total strain } \\
\text { isolates }\end{array}$ \\
\hline B1 & $\begin{array}{c}\text { FR4 (9); LR1 (11); } \\
\text { FR1 (17); NA1B (20) }\end{array}$ & 57 \\
\hline B2 & NA1A (19) & 19 \\
\hline B3 & LR1 (1) & 1 \\
\hline B4 & LR1 (1) & 1 \\
\hline B5 & BU1A (3); AL1B (11) & 14 \\
\hline B6 & BU1A (2); AS5A (13); & 16 \\
\hline & SA1B (1) & 1 \\
\hline B7 & BU1A (4) & 30 \\
\hline B8 & BU1A (3); AL4A (7); & 30 \\
\hline BU2A (17); AL1A (3) & BU1A (5) & 5 \\
\hline B10 & BU1A (1) & 1 \\
\hline B11 & BU1A (2) & 2 \\
\hline B12 & BU1A (3); BU1B (1) & 4 \\
\hline B13 & BU1A (2) & 2 \\
\hline B14 & BU1A (1) & 1 \\
\hline B15 & BU1A (1) & 1 \\
\hline B16 & BU1A (1) & 1 \\
\hline B17 & BU1A (1) & 1 \\
\hline B18 & BU1A (1) & 1 \\
\hline B19 & BU1B (9) & 9 \\
\hline B20 & AL4A (1) & 1 \\
\hline B21 & AL4A (1) & 1 \\
\hline B22 & AL4A (1) & 1 \\
\hline B23 & AL4A (1) & 1 \\
\hline B24 & AL4A (2) & 2 \\
\hline B25 & AL4A (1) & 1 \\
\hline B26 & BU2A (16) & 16 \\
\hline B27 & AL6A (1) & 1 \\
\hline B28 & AL7A (10) & 10 \\
\hline B29 & AL1A (17); AL1B (7) & 24 \\
\hline B30 & AL4A (1) & 1 \\
\hline & & \\
\hline & & \\
\hline
\end{tabular}

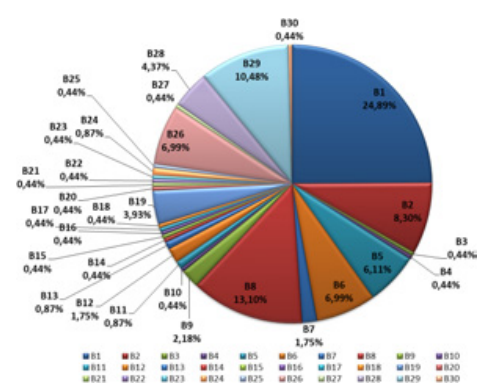

Figure 3. Frequencies of $S$. cerevisiae strains isolated from spontaneous fermentations of $V$. vinifera $L$. sylvestris grapes.

A wide strain diversity has been observed for S. cerevisiae wine yeasts isolated from wild grapes. 229 pure $S$. cerevisiae isolates from the end of spontaneous fermentations of $15 \mathrm{~V}$. vinifera ssp sylvestris grape samples were characterized by the profile of interdelta sequence amplification. According to the results, S. cerevisiae yeasts were classified in thirty genotypes or strains: B1 to B30 (Table 3). Collection of $S$. cerevisiae wine strains from natural fermentations has demonstrated the existence of such strong polymorphism within this specie [32].

Six strains: B1, B5, B6, B8, B12 and B29 were isolated from 2 up to 4 different locations. In some cases, the distance between sampling points where the same strain was found was more than $250 \mathrm{~km}$ (e.g. strain B6 isolated in grapes from BU1A and AS5A), proving an ecological niche in this area. In two locations, BU1A and AL4A, a high heterogeneity of strains was found: 14 and 8 different strains were found, respectively. Figure 3 shows the percentage of isolation of each $S$. cerevisiae strain. The most abundant strains were B1 (24.9\%), B8 (13.1\%), B29 $(10.5 \%), \mathrm{B} 2(8.3 \%), \mathrm{B} 6$ and B26 (7.0\% each one) and B5 $(6.1 \%)$.

At present, we are screening the different strains for their suitability for must fermentation (data not shown). Most of them are able to consume high amounts of sugar, achieving alcohol concentrations more than $14 \%$ vol, without increasing volatile acidity. Additional studies are being made on the ability of these strains to produce secondary products that contribute to the sensory properties of wines.

\section{Conclusion}

Yeasts are found throughout nature. However, they not occur randomly, but are found in specific habitats where different species form communities. In this sense, this is the first report about the native yeast diversity present in grapes of wild vines ( $V$. vinifera L., ssp sylvestris), a natural resource with oenological traits that might help to deal with the changes that climate change is producing in the vineyards. This work might provide supportive information for future studies on the contribution of these isolated yeast species to wine quality. We have identified some non-Saccharomyces species and several S. cerevisiae strains that have promising perspectives for its oenological use to improve wine quality. Further trials with these isolated strains, including the suitability of individuals and mixed cultures of non-Saccharomyces and S. cerevisiae strains at laboratory scale and in semi-pilot vinifications on different musts are in progress.

Spain's Ministry of Economy and Competitiveness, Project RTA2014-00016-C03-03, MINECO-INIA-CCAA.

\section{References}

[1] R. Mira de Orduña. Food Res. Int. 43, 1844-1855 (2010)

[2] A.D. Coulter, P.A. Henschke, C.A. Simos, I.S. Pretorius. Australian and New Zealand Wine Ind. J. 23, 26-30 (2008)

[3] F. Grassi, M. Labra, S. Imazio, R. Ocete Rubio, O. Failla, A. Scienza, F. Sala. Conservation Genetics 7, 837-845 (2006)

[4] M. De Andrés, A. Benito, G. Pérez-Rivera, R. Ocete, M.A. López, L. Gaforio, G. Muñoz, F. Cabello, J.M. Martínez-Zapater, R. Arroyo-García. Mol. Ecol. 21, 800-816 (2012)

[5] R. Ocete, R. Arroyo-García, M.L. Morales, M. Cantos, A. Gallardo, M.A. Pérez, I. Gómez, M.A. López. Vitis 50, 11-16 (2011)

[6] R. Ocete, M. Cantos, A. López-Gallardo, A. Pérez-Troncoso, M. Lara, O. Failla, F.J. Ferragut, J. Liñán. Caracterización y conservación del recurso fitogenético vid silvestre en Andalucía. Ed. Falcor. Sevilla (Spain) (2007) 
[7] R. Urso, K. Rantsiou, P. Dolci, L. Rolle, G. Comi, L. Cocolin. FEMS Yeast Res. 8, 1053-1062 (2008)A.

[8] OIV Resolution OIV-OENO 408 (2011)

[9] A. Querol, E. Barrio, D. Ramón. Syst. Appl. Microbiol. 15, 439-446 (1992)

[10] B. Esteve-Zarzoso, C. Belloch, F. Uruburu, A. Querol. Int. J. Syst. Bact. 49, 329-337 (1999)

[11] J.M Guillamón, J. Sabaté, E. Barrio, J. Cano, A. Querol. Arch. Microbiol. 169, 387-392 (1998)

[12] L. Granchi. M. Bosco, A. Messini, M. Vincenzini. J. Appl. Microbiol. 87, 949-956 (1999)

[13] J. Sabaté, J. Cano, B. Esteve-Zarzoso, J.Mo. Guillamón. Microbiol. Res. 157, 267-274 (2002)

[14] C.P. Kurtzman, C.J. Robnett. Antonie Van Leeuwenhoek 73, 337-371 (1998)

[15] C.X. Wang, Y.L. Liu. Food Microbio. 33,172-177 (2013)

[16] J.L. Legras, F. Karst. FEMS Microbiol. Lett. 221, 249-255 (2003)

[17] N. Cadez, P. Raspor, A.W.A.M. de Cock, T. Boekhout, M.Th. Smith. FEMS Yeast Res. 1, 279-289 (2002)

[18] N.P. Jolly, O.P.H. Augustyn, I.S. Pretorius. S.Afr.J.Enol.Vitic., 27, 15-39 (2005)

[19] P. Romano, C. Fiore, M. Paraggio, M. Caruso, A. Capece. Int. J. Food Microbiol. 86, 169-180 (2003)
[20] F. Viana, J.V. Gil, S. Vallés, P. Manzanares. Int. J. Food Microbiol.135, 68-74 (2009)

[21] F. Viana, C. Belloch, S. Vallés, P. Manzanares. Int. J. Food Microbiol. 151, 235-240 (2011)

[22] J. Lleixà, V. Martín, M.C. Portillo, F. Carrau, G. Beltran, A. Mas. Front. Microbiol. 7:338 (2016)

[23] H. Ertin, I. Campdell. J. Inst. Brew. 107, 207-215 (2001)

[24] J. Swangkeaw, S. Vichitphan, C.E. Butzke, K. Vichitphan. Ann. Microbiol.,59, 335-343 (2009)

[25] S.M. del Mónaco, M.E. Rodríguez, C.A. Lopes. Int. J. Food Microbiol. 230, 31-39 (2016)

[26] J. Garavaglia, R. C. de Souza-Schneider, S.D. Camargo-Mendes, J.E. Welke, C. AlcarazZini, E. Bastos-Caramao, P. Valente. Microbiol. Res. 173, 59-65 (2015)

[27] M. Gobbi, F. Comitini, P. Domizio, C. Romani, L. Lencioni, I. Mannazzu, M. Ciani. Food Microbiol. 33, 271-281 (2013)

[28] A. Benito, F. Calderón, F. Palomero, S. Benito. Molecules 20, 9510-9523 (2015)

[29] J.P. Morrissey, M.M.W. Etschmann, J. Schrader, G.M. de Billerbeck. Yeast 32, 3-16 (2015)

[30] C.P. Kurtzman, S. Droby. Syst. Appl. Microbiol. 24, 395-399 (2001)

[31] G.H. Fleet. FEMS Yeast Res. 8, 979-995 (2008)

[32] I.S. Pretorius. Yeast 16, 675-729 (2000) 